\title{
Hydro-dissection in a post hysterectomy female with dense labial fusion: a case report
}

\author{
Gayam S. ${ }^{1}$, Paul S. ${ }^{2}$, Lakshmi V.V.S. ${ }^{3}$, Geeta R. ${ }^{4}$, Asma T. ${ }^{5}$ \\ ${ }^{1}$ Dr. Susheela Gayam, DNB, DGO, ${ }^{2}$ Dr. Mary Sashikala, MD, DNB, ${ }^{3}$ Dr. Lakshmi VVSMS, FRCS, ${ }^{4}$ Dr. Geeta Rani, DNB, \\ ${ }^{5}$ Dr. Asma Tabasum, DGO, all authors are affiliated with Department of Obstetrics and Gynaecology, Vijay Marie Hospital \\ \& Educational Society, Hyderabad, India.
}

Corresponding Author: Dr. Susheela Gayam, DNB, DGO, Department of Obstetrics and Gynaecology, Vijay Marie Hospital \& Educational Society, Hyderabad, India. Email: sushgayam@yahoo.com

\begin{abstract}
Complete labial fusion or adhesion is a rare benign clinical condition seldom seen in adults. When the labia are especially completely fused, usually result in urinary problems like voiding difficulty, urinary retention, and urinary infection. Labial adhesions are generally caused by a combination of local inflammation, chronic infection and estrogen deficiency. We present a case of dense and complete labial fusion with voiding difficulty in a post hysterectomized woman. Patient underwent separation of the labial fusion by hydro-dissection followed by cystoscopic examination.
\end{abstract}

Key words: Dense labial fusion, Voiding difficulty, Hydro-dissection, Bladder trabeculations, Topical Estradiol therapy

\section{Introduction}

Labial adhesion is extremely rare in the reproductive age group due to abundance of estrogen. It is commonly described in prepubertal girls and less often reported in postmenopausal women and is associated with hypoestrogenic state, local inflammatory and irritative conditions, and vulvar dystrophies such as lichen sclerosis
[1]. Labial adhesion is defined as either partial or complete adherence of the labia minora or majora [2]. Here we present a case of almost complete labial adhesion with voiding difficulty in a 57 year old post hysterectomised woman and surgical management by hydro-dissection.

\section{Case Report}

A 57 years old woman, P2L2who underwent hysterectomy 10 years ago, presented with difficulty in voiding urine for3 months. There was no history suggestive of retention of urine, burning micturition, dribbling of urine or any history of bleeding per vagina or discharge per vagina. The couple stopped having coitus since the time of hysterectomy. She was a known case of hypothyroidism since 3 years and was on Thyroxine $25 \mu \mathrm{g}$. There was no history of diabetes mellitus or hypertension.

On general physical examination she was moderately built and well nourished. The vital parameters were within normal limits. The systemic examination of cardiovascular, respiratory, and abdomen showed no abnormality. The local examination showed densely fused labia majora and minora from anterior to posterior, all along the full length in the midline up to the clitoris proximally and fouchete distally except for a small opening seen at the distal end. Urethral orifice and the vaginal opening were not spotted. Per rectal examination showed no evidence of any collection. Ultrasound examination confirmed post hysterectomy status and the ovaries were not visualised. All other investigations were within normal limits. CUE and urine culture and sensitivity were normal.

Patient underwent EUA (Examination under anesthesia), Labial adhenolysis by hydro-dissection followed by Cystoscopy. The following findings were noted - both thelabia majora and minor were fused densely in the midline extending from clitoris to the fourchette (Figure 1) with a small opening at the fouchettethat allowed the passage of an artery forceps for bout $3-4 \mathrm{~cm}$

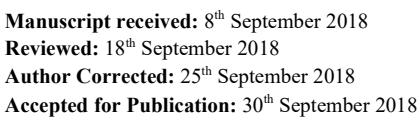




\section{Case Report}

length and the upper end was blind(Fig 2); urethral and introital openings were not visualised. Since the adhesions were dense, manual separation was not possible. Therefore Hydro-dissection was performed by injecting normal saline with adrenalineinto the fused area (Figure 3) and a small incision was then made in the centre and a gentle traction applied on both the labia and separated them without much difficulty. Following the labial separation, per speculum examination revealed normal urethral opening, vagina and the vault (Figures 4-6).

Cystoscopy was carried out as patient had voiding difficulty and it showed marked bladder trabeculations secondary to distal obstruction of urinary flow (Figure 7). Both the ureteric orifices were visualized and were normal and urinary efflux was seen (Figures 8-9). Post operatively the patient was catheterized (Figure 10) and vaginal pack was kept for 24 hours and advised topical application of antibiotic ointment and estradiol cream to prevent re-adhesion. Patient was discharged on day 4 of surgery and was recommended to continue to use topical estradiol cream for six weeks. She was reviewed on regular basis for 6 months. She had no voiding difficulty and the raw areas healed well.
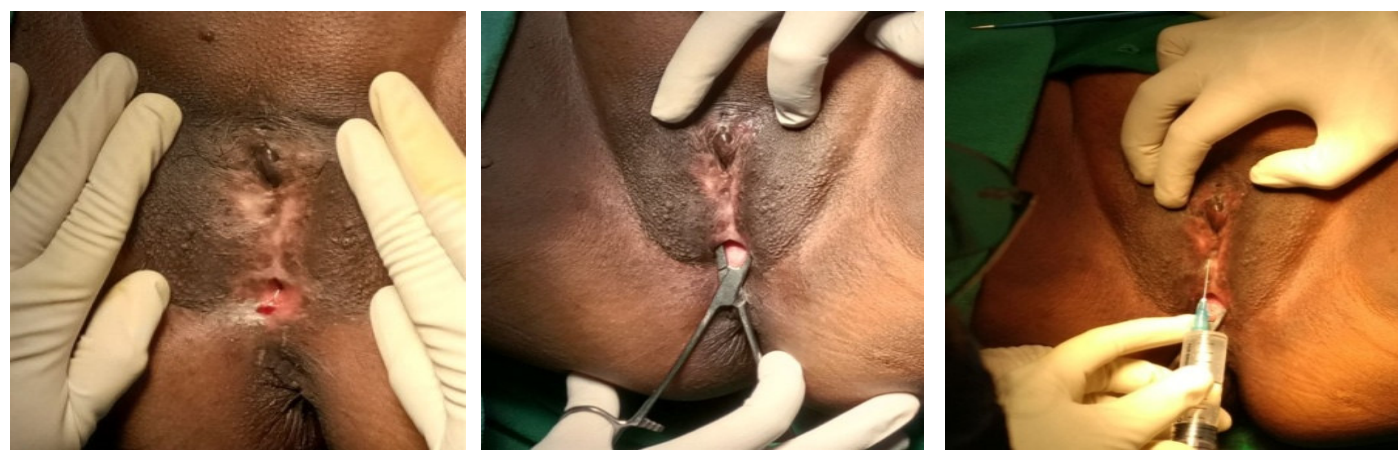

Figure1: Preoperative: Fused labia in the midline with obliteration of vaginal introitus and urethral meatus Figure2: A Smalll opening at the fourchette shown by the placement of artery forceps

Figure3: Instillation of Normal saline for Hydro-dissection
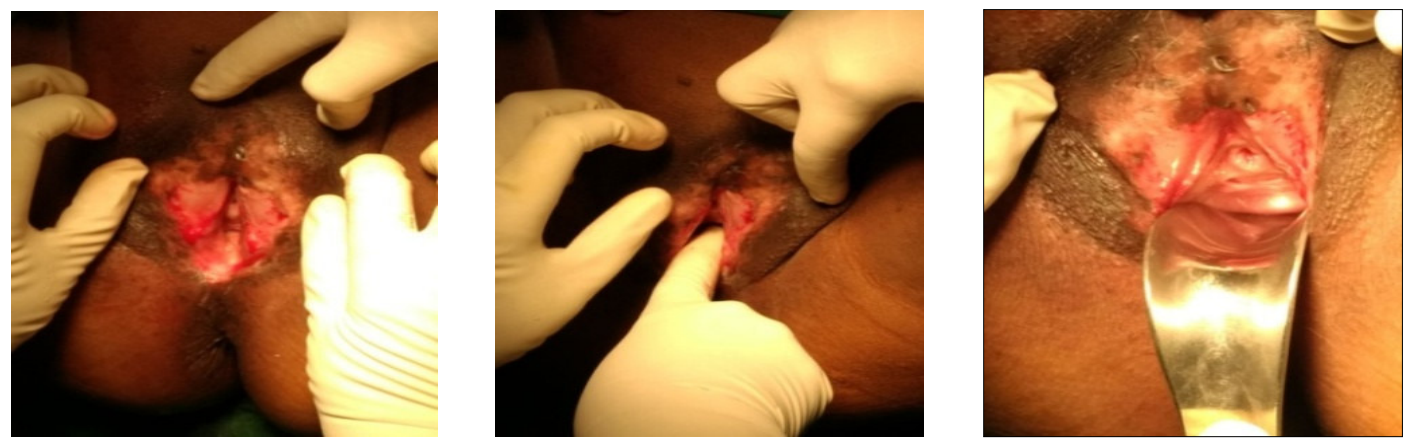

Figures 4-6: Postoperative view of the introitus following separation of labial adhesions; showing raw area, digital examination of vagina and per spculum examination.
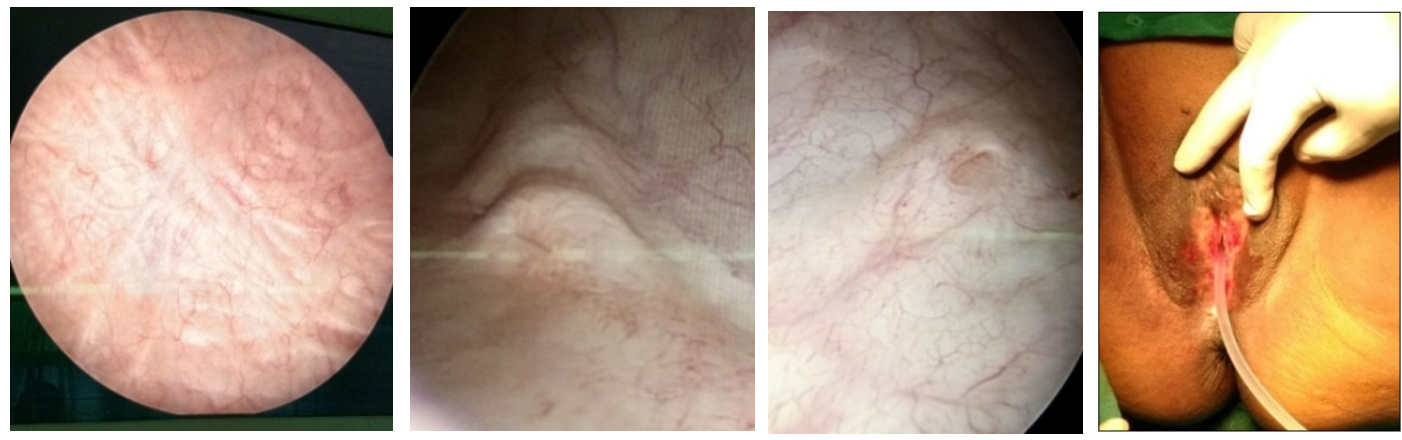

Figure 7: Cystoscopic examination showing increased bladder trabeculations.

Figures 8-9: Cystoscopic examination showing right and left ureteral openings.

Figure 10: Post op insertion of Foley catheter into the urethral meatus. 


\section{Case Report}

\section{Discussion}

Labial synechia are usually caused by a combination of local inflammation, chronic infection and estrogen deficiency. This condition is not life threatening, but severe cases usually result in urinary problems. Labial fusion is diagnosed by visual inspection, and the clinical complications associated with labial fusion are usually minor. However, urinary tract infection or hydronephrosis can result from disturbances in urination [3, 4]. Surgical adhesiolysisis needed if a patient complains of urination disorders or the degree of labial fusion is severe. These adhesions are usually superficial, but sometimes they can be dense involving the clitoris, thus making it difficult to distinguish the precise anatomic location [5]. In the present case, surgical intervention with hydro-dissection was carried out as a safe measure since the manual separation was not possible due to the dense labial adhesions and obscured anatomy.

Patients with a suspected bladder outlet obstruction had a significantly higher incidence of bladder trabeculation. Bladder trabeculation, which is detected by a cystoscope, is the secondary result of a bladder outlet obstruction and is known to be caused by morphological and histological changes due to hypertrophy and hyperplasia of the bladder muscle and the infiltration of the connective tissue [6]. Some studies have suggested that bladder trabeculation is generated by the aging process. However, a principal pathological mechanism was reported to be connected with changes of the bladder muscle in order to compensate for the increased urethral resistance in the lower bladder, which is caused by physiological or anatomical reasons such as a bladder outlet obstruction [6]. In the present case Cystoscopy was done as patient presented with voiding difficulty and it showed increased bladder trabeculations suggesting bladder outlet obstruction. Postoperative estrogen cream helps prevent recurrences and repeat surgery [2].

\section{Conclusion}

In conclusion, we high light that when a patient presents with dense labial adhesions, obscured anatomy and voiding difficulties, hydro-dissection can be used as a safe surgical intervention for adhenolysis. Following labial separation cystoscopic examination of the bladder will help in ruling out infection and confirm distal obstruction by the presence of bladder trabeculations. Topical application of estradiol cream and emollients like vaseline will help prevent reformation of adhesions. Surgical approach especially hydro-dissection is a preferred choice for this case as the dense synechia caused voiding problems and distorted anatomy.

Consent for Publication- Written informed consent was obtained from the patient for publication of this case report and the accompanying images. A copy of the written consent is available for review by the Editor-inChief.

Competing Interests- The authors declare that there is no conflict of interests regarding the publication of this paper.

\section{Funding: Nil, Conflict of interest: Nil Permission from IRB: Yes}

\section{References}

1. Muppala H, Meskhi A. Voiding dysfunction due to long-standing labial fusion in an elderly woman: a case report.IntUrogynecolJPelvicFloorDysfunct.2009;20:251-2

2. Sentürk S, Ustüner P, Balık G, et al. Labial adhesion with acute urinary retention secondary to vaginitis. Case Rep Obstet Gynecol. 2014;2014:259072. doi: 10.1155/ 2014/ 259072. Epub 2014 Jul 7.

3.Azarfar A,RavanshadY,Bagheri S,et al. Labial adhesion and bacteriuria.J TurkGerGynecolAssoc.2015 Jun1;16(2): 68-9.doi:10.5152/jtgga.2015.15222.eCollection 2015.

4. Melek E, Kilicbay F, Sarikas NG, Bayazit AK. Labial adhesion and urinary tract problems: The importance of genital examination. J Pediatr Urol. 2016; 12:111. doi: 10. 1016/j.jpurol.2015.10.002.

5. Kumagai Y, Toyoshima M, Kudo K, et al. Endoscopic examination of labial fusion in a postmenopausal woman: a case report. J Med Case Rep. 2018 Feb 2;12(1):26. doi: 10. 1186/s13256-018-1568-4.

6. Bai SW, Park SH, Chung DJ, et al. The Significance of Bladder Trabeculation in the Female Lower Urinary System: An Objective Evaluation by Urodynamic Studies. Yonsei Medical Journal. 2005; 46(5):673-678. doi:10. 3349 /ymj. 2005.46.5.673.

\section{How to cite this article?}

Gayam S, Paul S, Lakshmi V.V.S, Geeta R, Asma T. Hydro-dissection in a post hysterectomy female with dense labial fusion: a case report.Obs Rev:J obstet Gynecol 2018;4(3):46-48.doi: 10.17511/joog.2018.i03.01. 